\title{
Lessons from BRCA: \\ The Tubal Fimbria Emerges as an Origin for Pelvic Serous Cancer
}

\author{
Christopher P. Crum, MD; Ronny Drapkin, MD, PhD; David Kindelberger, MD; \\ Fabiola Medeiros, MD; Alexander Miron, PhD; and Yonghee Lee, MD
}

\begin{abstract}
Ovarian epithelial cancer is diagnosed in approximately 25,000 women yearly in the United States, accounting for approximately 12,500 deaths. Of these tumors, serous cancer is the most lethal, due to its capacity to spread beyond the reproductive tract and involve the peritoneal surfaces or distant organs. Conventional classification systems designate tumor origins principally on the location of the largest tumor. However, despite the fact that the largest tumors typically involve the ovaries, demonstrations of a precise starting point for these tumors, including precursor lesions, have been inconsistent. In recent years, a major effort to prevent serous cancer in genetically susceptible women with mutations in BRCAI or BRCA2 has spawned the practice of prophylactic salpingo-oophorectomy. This practice has surprisingly revealed that many early cancers in these women arise in the fallopian tube, and further studies have pinpointed the distal (fimbrial) portion as the most common site of origin. Emerging studies that carefully examine the fallopian tubes suggest a high frequency of early cancer in the fimbria in unselected women with ovarian and peritoneal serous carcinoma, raising the distinct possibility that a significant proportion of these tumors have a fimbrial origin. The evidence for these discoveries and their relevance to serous cancer classification, early detection and prevention are addressed in this review. A model for pelvic serous cancer is proposed that takes into account five distinct variables which ultimately impact on origin and tumor distribution: $(I)$ location of target epithelium, (2) genotoxic stress, (3) type of epithelium, (4) mitigating genetic factors, and (5) tumor spread pattern. Ultimately, this model illustrates the importance of identifying cancer precursors, inasmuch as these entities are useful as both surrogate endpoints for their respective malignancies in epidemiologic studies and natural targets for cancer prevention.
\end{abstract}

Keywords: BRCA, Fallopian tube neoplasms; Fimbria; Ovarian neoplasms; p53; SEE-FIM; Serous carcinoma

$\mathrm{O}$ varian epithelial cancer is diagnosed in approximately 25,000 women yearly in the United States with approximately one half dying of their disease. ${ }^{1}$ It typically afflicts women near to menopause or postmenopause and peaks in the sixth to seventh decades of life. Surgery and chemotherapy will produce a complete response in $70 \%$ of patients; however, relapse rates are high, often occurring after a relatively brief respite. Overall long-term survival, but not cure, rates have only begun to improve measurably with the advent of taxol chemotherapy and intraperitoneal routes of instillation. However, the incremental gain in disease-free survival seen with these new therapies is only a few months. ${ }^{2}$

Reprint Requests: Christopher P. Crum, MD, Department of Pathology, Brigham and Women's Hospital, 75 Francis Street, Boston, MA 02115, Tel: 6|7-732-548I, Fax: 617-264-5I25, Email: ccrum@partners.org

Approximately $10 \%$ of women with ovarian cancer have a positive family history and most have an inherited heterozygous mutation in the BRCA1 or BRCA2 gene. Women with a hereditary predisposition for ovarian cancer develop the disease on average a decade earlier., ${ }^{3,4}$ The lifetime risk of ovarian cancer in women with mutations in BRCA1 or BRCA2 (BRCA+) is estimated to be as high as $56 \%$ and $27 \%$, respectively, ${ }^{5}$ with risk of clinical cancer increasing in the fifth decade. ${ }^{3-5}$ Management of this group centers on removal of the fallopian tubes and ovaries prior to the onset of clinical cancer. Less than $10 \%$ of these specimens are determined to contain occult cancer, and many do not relapse. Cancer-free rates for those undergoing

Received: May 15, 2006
Revised: September 30, 2006

Accepted: October 19, 2006

DOI: $10.3121 / \mathrm{cmr} \cdot 2007.702$
Grant Support: National Cancer Institute (P50 CA 10500), the Francis Ward Paine and TSA Pemberton Funds of the Division of Women's and Perinatal Pathology, Brigham and Women's Hospital, and Columbia Hospital for Women Research Foundation, Washington, DC. 

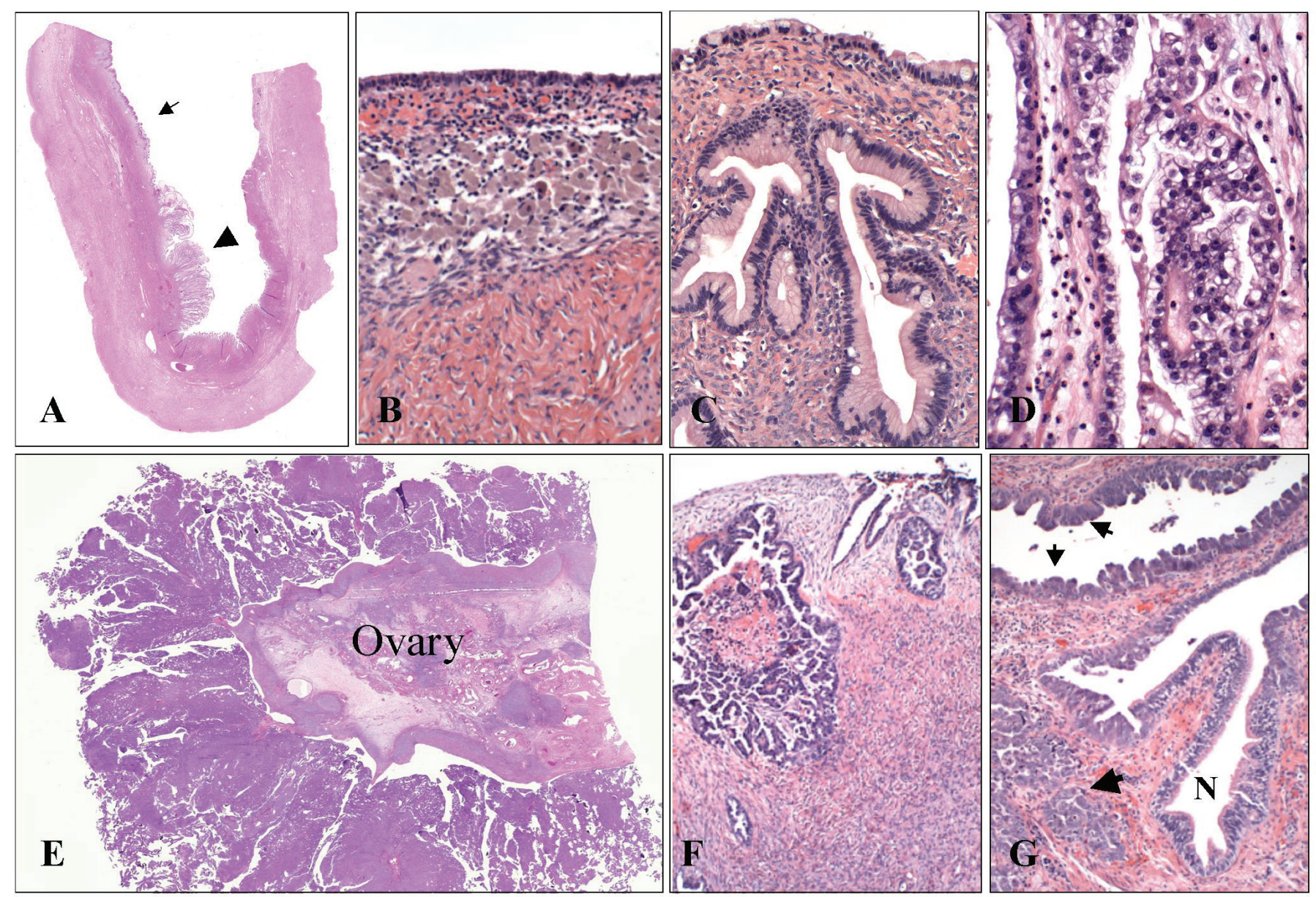

Figure 1. Example of an ovarian tumor with a broad phenotypic range arising by a sequence of genetic events (so-called Type I tumor). ${ }^{18-20}$ (A) A large ovarian cyst contains evidence of preexisting endometriosis (small arrow) in which a clear cell carcinoma has developed (large arrow). Elsewhere in the cyst, (B) benign endometrial epithelium is juxtaposed with (C) a mucinous cystadenoma and (D) the malignant tumor. Type II high-grade serous carcinoma of presumably short duration frequently involve $\left(\mathrm{E}\right.$ and $\mathrm{F}$ ) the ovarian surface. ${ }^{18}$ However, many are associated with $(\mathrm{G})$ an early carcinoma in the distal fallopian tube. ${ }^{50,53}$

prophylactic surgery are nearly $96 \%$ versus $69 \%$ for those managed by close surveillance alone, ${ }^{6}$ demonstrating the overall benefit of prophylactic surgery for this population. The much lower success rate for those managed by close surveillance alone testifies to the aggressive properties of pelvic serous cancer, which spreads rapidly following onset (figure 1), in this population. Overall, serous tumors (borderline and malignant) account for nearly half of all ovarian epithelial tumors and are more likely to be advanced stage when diagnosed, relative to endometrioid and mucinous carcinomas. ${ }^{7}$

\section{Early Detection of Ovarian Cancer}

The discovery of CA125 two decades ago initiated a sustained effort to identify and employ biomarkers in the detection of ovarian cancer. ${ }^{8}$ These studies, which often include a second parameter such as ultrasound, have improved the sensitivity and specificity of this cancer screening approach. CA125 is used primarily to monitor patients following treatment and is used as a surrogate for both response to treatment and cancer recurrence. ${ }^{9}$ Other biomarkers, including human kallikrein,
HE4 and osteopontin, are under investigation and large screening trials are underway in the United Kingdom. ${ }^{10-13}$

Efforts to use multiple biomarkers, although encouraging, have met with mixed results. One study comparing the serologic detection of multiple biomarkers demonstrated comparable sensitivity (79\% and $82 \%$ ) and an improvement in specificity from $68 \%$ to $88 \%$. Subsequently, the authors prospectively screened healthy women, producing a specificity of $100 \%$ for multiple markers compared to $94.8 \%$ for CA125II alone. ${ }^{14}$

Despite the relatively high sensitivity and specificity of some markers under evaluation, it is important to emphasize that a test with a $99 \%$ specificity will still necessitate as many as 25 abdominal surgeries to uncover one case of cancer. The tendency for serous ovarian cancers to spread early underscores the problems that have been encountered with the use of biomarkers to detect and manage this disease. The central argument is that early detection of the more lethal tumors, serous carcinomas, is not practical. ${ }^{15}$ 


\section{Origin of Ovarian Cancer}

The origin of ovarian cancer is a function of two distinct but interrelated variables: cell of origin and molecular pathway. Ovarian carcinomas are by composition epithelial. However, because the ovarian cortical stroma does not harbor epithelial cells at birth, the process of epithelial development presumably occurs over time. Ovarian cancer has traditionally been presumed to arise in the ovarian surface epithelium (OSE) or in derived ovarian cortical epithelial inclusions, which exhibit the morphologic features of reproductive tract (Mullerian) epithelium. ${ }^{16}$ These Mullerian inclusions are acquired during reproductive life. Three possible mechanisms for their development include (1) epithelium from the distal fallopian tube (endosalpingiosis) by exfoliation or tubal-ovarian adhesions, (2) invagination and incorporation of the OSE into the cortex during repair of ovulation, and (3) implantation of cells from the endometrium via retrograde menstruation (endometriosis). ${ }^{17}$

The second variable that is integral to ovarian carcinogenesis is the molecular pathway(s) involved, for which two general categories have been defined. The first is the Type I pathway, which involves mutations in PTEN, K-ras, B-RAF and beta-catenin. This pathway typically involves a multi-step process, such that these tumors may exhibit a spectrum from benign to malignant. Examples include endometriosis and endometrioid carcinoma, or a combination of benign, borderline, and malignant serous or mucinous ovarian tumors. ${ }^{18}$ This gradual process of malignant transformation occurs within the substance of the ovary (either in inclusion cysts or endometriosis) and does not usually extend beyond the ovary until late in its course. This is supported by recent mouse models exploiting sequential inactivation of the K-ras and PTEN genes. ${ }^{19}$ Based on recent studies, the acquisition of Mullerian epithelial phenotypes in the OSE is a function of the HOX gene expression linked to these cells. ${ }^{20}$

The second pathway appears to develop quickly and includes mutations in the p53 tumor suppressor gene. The prototypical tumor is a high-grade serous carcinoma, which appears to develop rapidly and, invariably, involves the ovarian surface. This characterizes the tumors seen in the BRCA+ population, but is similar in tumors that are not associated with a family history, such as sporadic serous carcinomas (figure 1). Tumors of this type express a phenotype that most closely resembles fallopian tube mucosa and contain p53 mutations. ${ }^{21,22}$

The OSE/Mullerian inclusion theory is the most enduring and explains some ovarian tumors, while the magnitude of the role of OSE in this process remains controversial. The reason is the consistent lack of irrefutable morphologic evidence of an ovarian precursor lesion on the surface epithelium. ${ }^{23}$ One case report identified intraepithelial carcinoma in inclusion cysts. ${ }^{24}$ Another reported dysplastic changes in eight ovarian inclusion cysts from 13 cases of ovarian cancer. Of these 13 cases, five were also immunostained for p53; however, the molecular basis for the p53 staining was not determined. ${ }^{25}$ Another study characterized early ovarian cancers from a large pathology consultation practice which identified 14 small serous carcinomas. Although presumed to have originated in the ovary (based on their small size), most were not associated with a candidate precursor lesion. ${ }^{26}$ Some studies have noted increased expression of cancer-associated biomarkers in the OSE of women with BRCA mutations or ovarian cancer, 27 fostering the concept of progressive dysplastic changes in the OSE in these groups, while others have found the reverse. Another study of ovaries prophylactically removed from women at risk for ovarian cancer did not identify evidence of a precursor lesion in the ovarian cortex. $^{28}$

Although the precise nature of ovarian intraepithelial precursors to ovarian serous carcinoma remain unresolved, the significance of such an entity has not been lost on those dedicated to preventing this disease. ${ }^{29}$ For example, an excellent model for cancer pathogenesis and prevention is the cervical cancer precursor (i.e., cervical intraepithelial neoplasia), which has been established as a necessary prerequisite to cervical cancer development. ${ }^{30}$ This precursor was linked to cancer by the presence of the most prevalent cancer-associated human papilloma virus (HPV), type 16.31 Subsequent viral particle (pseudovirion) vaccines generated in vitro to this HPV type have been effective in preventing the emergence of HPV-16 associated precursor lesions. ${ }^{32}$ It is therefore assumed that these vaccines will be effective in preventing cervical cancer, and is the basis for continued trials and efforts to bring this vaccine to the public. By analogy, the identification of a precursor lesion for pelvic serous cancer has broad implications to not only early detection and prevention but also formulating a cogent pathway for tumor development.

\section{Lessons from the BRCA Population}

As a group, women with BRCA1 and BRCA2 mutations have provided opportunities to address the feasibility of prophylactic surgery in the prevention of pelvic serous carcinoma. $^{33}$ Rebbeck et al $^{34}$ identified just two peritoneal cancers in 253 women who were cancer-free at the time of prophylactic surgery. In contrast, 58 of 292 women followed without surgery developed a malignancy. ${ }^{34}$ In addition to the obvious benefit gained in risk reduction, prophylactic surgery in these women has afforded the unique opportunity to observe the early events of serous carcinogenesis, an advantage rarely experienced with sporadic cancers that present at high stage. From these studies, two possibly related observations shed light on the pathogenesis of pelvic serous cancer in this population. The first was that despite prophylactic surgery and the report of normal tubes and ovaries, a small subset of women subsequently returned with pelvic serous carcinoma. 33,34 This observation was viewed as a strong endorsement for a secondary Mullerian system, presumably arising by Mullerian metaplasia of the pelvic surfaces or implants of transported Mullerian epithelium 
Table 1. Frequency of fallopian tube carcinoma in women with BRCA mutations.

\begin{tabular}{|c|c|c|c|c|}
\hline Authors & Number & Cancer (\%) & Fallopian tube (\%) & Comments \\
\hline Finch $^{43}$ & 159 & $7(4.4)$ & $6(84)$ & \\
\hline Kauff ${ }^{33}$ & 98 & 1 & 0 & One classified as peritoneal \\
\hline Medeiros $^{50}$ & 13 & $5(5)$ & $5(100)$ & Four cases involved fimbria \\
\hline Total & 481 & 28 & 15 & \\
\hline
\end{tabular}

from the uterus or fallopian tubes. There is no argument that such a system exists in some form; however, assignment of pelvic serous carcinomas to a peritoneal origin was based principally on the lack of other candidate primary sites.

The second was the increasing attention to the fallopian tube. Estimates of fallopian tube cancer annual incidence in the United States are approximately one-fiftieth (0.3-15 per $100,000)$ of ovarian cancer. ${ }^{35}$ This is due in part to at least three factors. The first, and most obvious, is the criteria for fallopian tube cancer which requires a dominant mass in the fallopian tube and evidence of an early (i.e., intraepithelial) carcinoma in the endosalpinx to explain an origin at that site. ${ }^{36}$ Thus, a tumor arising in the tube could be arbitrarily classified as peritoneal or ovarian if the tumor bulk in the latter site(s) was more substantial. Second, the fallopian tubes are not routinely examined completely in cases of pelvic serous cancer, in which case a tubal source might be overlooked. Third, there is some conceptual inertia in any field that must be overcome before a significant shift in thinking is to occur. Despite these factors, in the past 10 years, the fallopian tube has been emerging as a candidate site for serous carcinogenesis, principally because it was being identified in its earliest form when the origin is indisputable. ${ }^{37}$ Beginning in the late 1990s, investigators began identifying early serous carcinomas of the fallopian tube in prophylactic salpingo-oophorectomies from women with BRCA mutations (BRCA +$).{ }^{38-40}$ This finding necessitated a shift in management with emphasis on removing both tubes and ovaries from these patients. Since then, early serous carcinoma has been diagnosed in the fallopian tubes from approximately $2 \%$ to $10 \%$ of $\mathrm{BRCA}+$ patients undergoing prophylactic bilateral salpingo-oophorectomy (table 1). ${ }^{42,43}$ Reported differences in prevalence may be influenced by study design, subjects age and the extent to which the tubes were sampled. Depending on the study, from $40 \%$ to $100 \%$ of tumors discovered in these patients were located in the fallopian tube. In some of these cases, the carcinomas were detected sufficiently early that they were confined to the endosalpinx epithelium (tubal intraepithelial carcinoma, TIC) and their origin in the fallopian tube could not be disputed (figure 2). What is immediately appreciated from these reports is that the frequency of early tubal carcinoma in prophylactic salpingo-oophorectomy specimens contrasts sharply with the reported frequency of tubal cancer relative to ovarian cancer. ${ }^{37}$ It could be argued that BRCA patients are more likely to harbor tubal cancers; one study noted that over $40 \%$ of their patients with tubal cancer were $\mathrm{BRCA}+{ }^{44}$ However, other series of tubal cancers have not shown a difference in frequency of BRCA mutations between tubal and ovarian cancers. ${ }^{45-47}$

\section{The Fimbria as a Preferred Site for Early Serous Carcinomas}

Because the fallopian tube is a major site of early cancer development in a group traditionally at risk for ovarian cancer $(\mathrm{BRCA}+)$, investigators must revise their perceptions of the origin(s) of pelvic serous cancer. Although it is conceivable that $\mathrm{BRCA}+$ women are more prone to fallopian tube carcinomas than those without a family history, investigators hypothesized that the high frequency of tubal carcinoma in the BRCA+ population signified the tube as a more frequent site of origin than previously suspected. The most familiar presentation of tubal cancer is a distended organ with a large tumor, often centrally located. However, because this presentation is decidedly uncommon, we hypothesized that the distal fallopian tube (fimbria) was a more likely site of initiation. Tumors arising in this region would be less commonly recognized as primary tubal carcinomas because they would either spread rapidly to other sites or become complexed with the ovary and, therefore, not appreciated as the site of origin. In fact, reports of fimbrial carcinomas are uncommon, a fact that might reflect the practice of selective pathologic sampling of the fallopian tube in cases of presumed ovarian cancer. ${ }^{48}$ Moreover, the fimbria is a unique region that has a large surface area, is exposed to biologic events that also impact on the ovarian surface, and is a junction between the mesothelium and Mullerian epithelium.

Complete histologic sampling of the fallopian tubes and ovaries is required in prophylactic salpingo-oophorectomies because the tumors can be extremely small. To ensure a thorough examination of the entire tube, including the fimbria, we developed a protocol for sectioning and 


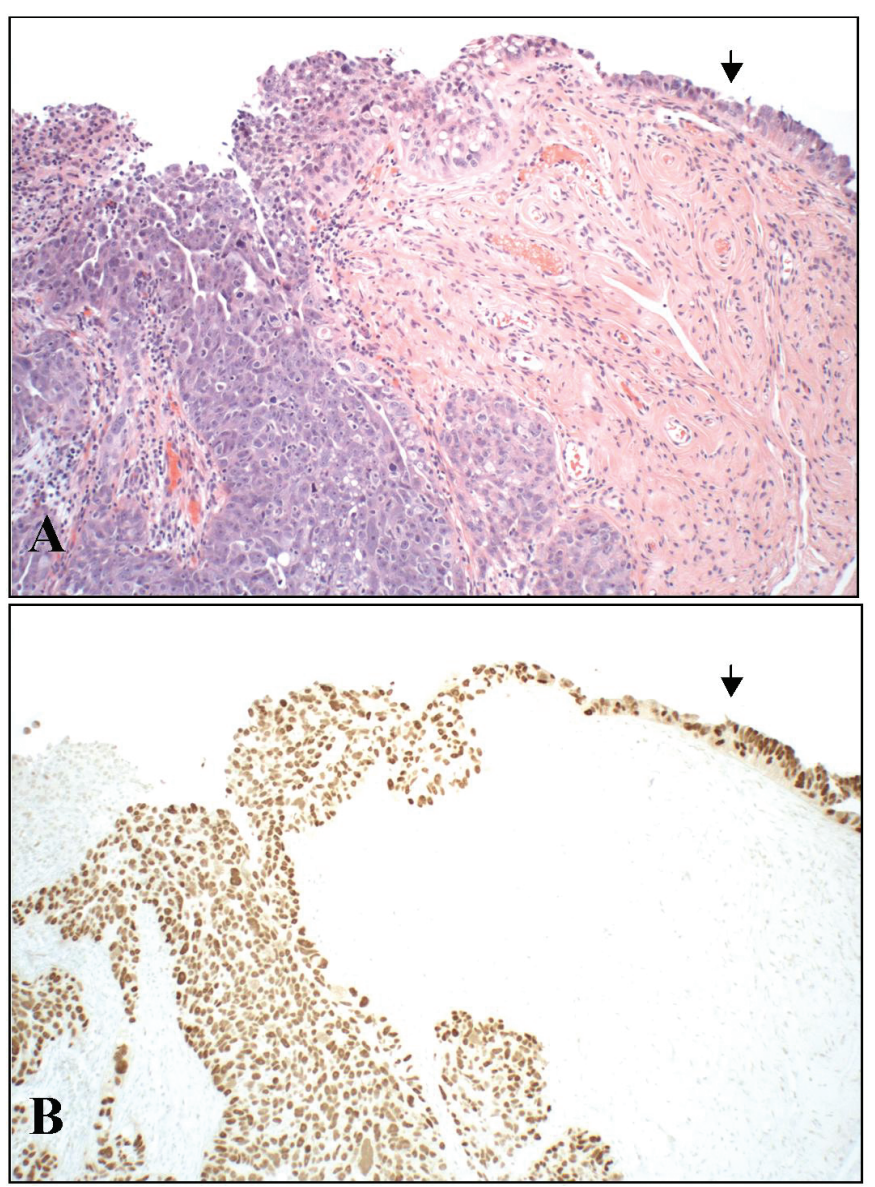

Figure 2. Appearance of early serous cancer (intraepithelial carcinoma, arrow) arising in the fimbria of a BRCA+ woman and merging with invasion ( $A$, left). These tumors are typically (but not necessarily) strongly positive for p53 protein by immunohistochemistry $(B)$, reflecting in most cases a p53 mutation.

extensively examining the fimbrial end (SEE-FIM), and implemented this protocol in salpingo-oophorectomies from both BRCA+ women and unselected pelvic serous carcinomas. ${ }^{49}$ In an analysis of over 100 consecutive prophylactic specimens from this population, a proportion of which have been analyzed by SEE-FIM, we have identified five tumors. Four of these tumors arose from the fimbria and one was immediately proximal in the ampullary region (figure 2) (M. Callahan, personal communication). ${ }^{50,51}$ Two other studies showed similar findings, ${ }^{43,44}$ one of which showed that the distal fallopian tube is the preferred site of early tubal cancer irrespective of BRCA status. ${ }^{44}$ The logical question in the face of this information is whether the distal fallopian tube is involved in sporadic ovarian and peritoneal serous carcinomas.

\section{The Fimbria in Unselected Women with Pelvic Serous Cancer}

Tubal involvement in ovarian cancer has been estimated at approximately $15 \%$. However, as mentioned above, pathologists do not typically examine the tube in detail. Most of the tumor in such cases is located on the serosal surface of the mesosalpinx, reflecting the tendency of serous carcinomas to involve peritoneal-lined surfaces. Once this is documented, the status of the endosalpinx is effectively moot in terms of clinical management. Thus, early tubal cancer (TIC) of the endosalpinx is uncommonly reported in association with ovarian cancer or, if observed, is attributed to mucosal implants from an ovarian source or a separate primary site. ${ }^{52}$

To determine the proportion of non-familial serous carcinomas associated with early fallopian tube carcinoma, we examined a consecutive series of pelvic serous carcinomas using the SEE-FIM protocol. We found that approximately $70 \%$ of consecutive pelvic serous carcinomas involve the endosalpinx. Interestingly, nearly one-half of tumors classified as ovarian serous carcinoma co-existed with an early tubal cancer (TIC)..$^{53}$ To determine if the tubal and ovarian tumors were genetically related, we analyzed both for mutations in the tumor suppressor gene p53, alterations of which are integral to serous carcinogenesis. In a small series ( 5 cases), both the tubal and ovarian cancers shared identical mutations. In addition, of seven tumors classified as a primary peritoneal serous carcinoma, six also involved the endosalpinx and four demonstrated clear evidence of an early cancer arising in the fallopian tube. ${ }^{53}$ Based on these findings, it is conceivable that many so-called peritoneal and ovarian serous carcinomas originate in the inner lining of the distal fallopian tube.

Additional support for the tube as an origin for serous carcinomas has been addressed in prior studies, including those showing that the gene expression profile of serous neoplasia closely resembles that of the normal fallopian tube. ${ }^{54}$

Potential Early Precursors to Pelvic Serous Carcinoma Intraepithelial carcinoma of the fallopian tube can be difficult to recognize if the lesion is very small, in part because these lesions can be confined to only a few cell layers. Moreover, nuclear stratification is common in benign salpingeal mucosa. ${ }^{50}$ In our efforts to detect early serous carcinomas in

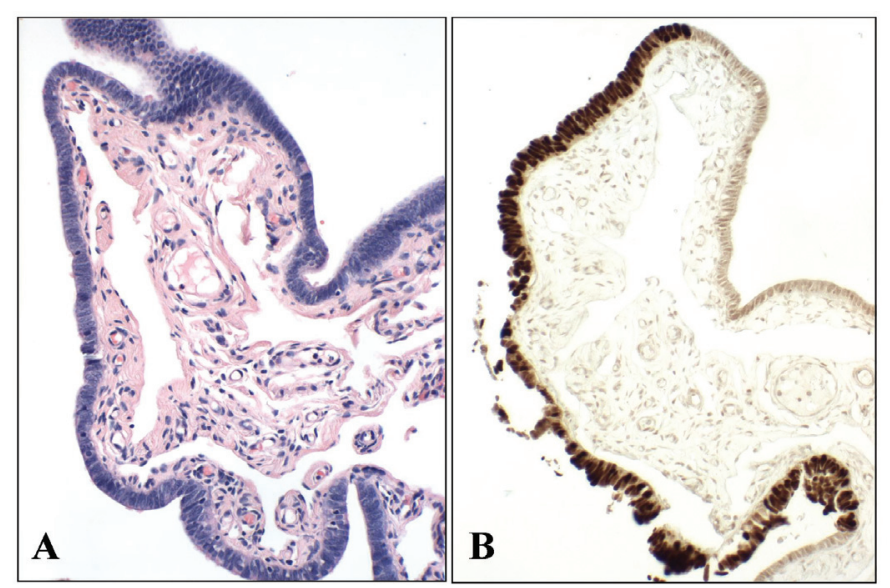

Figure 3. Normal appearing salpingeal mucosa (A) with strong p53 positivity (B). p53 signatures share many similarities (e.g., cell type, location, p53 mutations) with serous carcinoma. ${ }^{55}$ 


\section{Fimbria}

\section{Ovary}

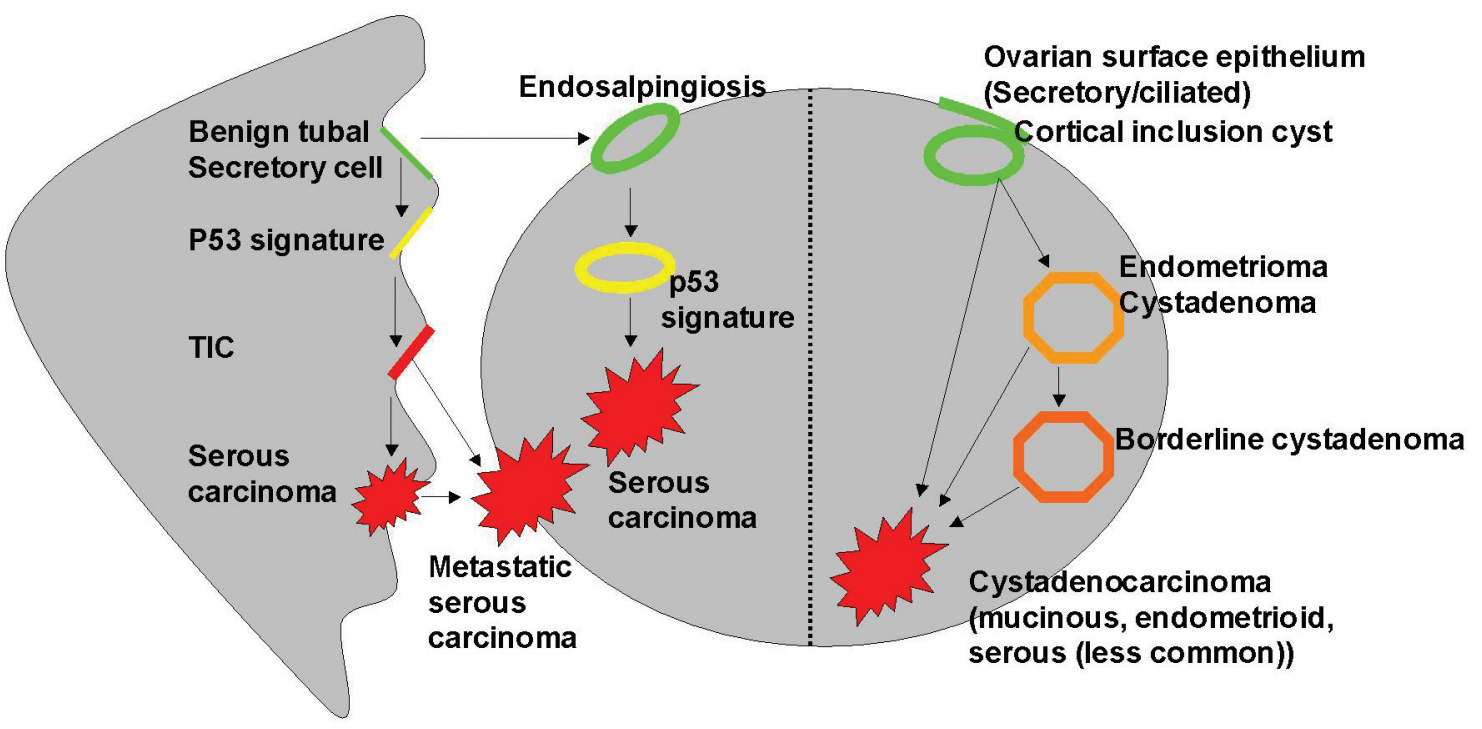

Figure 4. This proposed model integrates the molecular models of ovarian cancer with the fimbrial model of serous carcinogenesis, further taking into account cell of origin and pattern of spread. ${ }^{18,50,53,55}$ The pathway on the right originates in the ovarian epithelial inclusions, giving rise to most Type I neoplasms (and less commonly, serous carcinomas) by step-wise mutations, and includes endometriosis, benign and borderline cystadenomas. The pathway on the left would arise in either the fimbrial mucosa or tubal epithelium that has become adherent to the ovarian cortex (endosalpingiosis). In this pathway, the initial step consists of a p53 mutation following genotoxic injury (the "p53 signature"). In the fimbria, a tubal intraepithelial carcinoma (TIC) develops in some instances and may invade locally or exfoliate to other peritoneal (e.g., ovary or pelvis) surfaces. Depending on the location and rate of tumor growth, the tumor might be presumed to originate from the tube, ovary, or peritoneum. This would explain many, but not all, high grade (Type II) tumors.

$\mathrm{BRCA}+$ women, we employed a commonly used immunohistochemical stain for the p53 protein. This protein is over-accumulated in serous carcinoma and registers as a strongly positive intranuclear signal. We found that early serous carcinomas of the tube also stained strongly for p53 facilitating detection of difficult cases. However, a significant pitfall in interpretation stems from the fact that occasionally foci of benign-appearing mucosa also stain strongly for this marker. We termed these foci "p53 signatures."51 Because of this, we routinely employ both MIB-1 (a proliferative marker) and p53 immunostaining as adjuncts to histopathologic evaluation when discriminating neoplastic from non-neoplastic p53 positive epithelium.

Although a potential pitfall in the interpretation of early fallopian tube neoplasia, p53 signatures may hold considerable promise. We have found that these signatures are most commonly present in the distal tube and predominate in a specific cell type that is the presumed cell of origin for tubal serous carcinoma (i.e., secretory). Currently, studies are in progress to determine if p53 signatures exhibit other properties that fulfill the requirement of an early precursor to serous carcinoma (figure 3). ${ }^{55}$

\section{An Emerging Model for the Pathogenesis of Pelvic Serous Carcinoma}

In light of the evidence presented above, the following model of pelvic serous carcinogenesis is proposed that provides for two distinct pathways of tumor development (figure 4 and table 2). The first pathway consists of a traditionally accepted route, in which the Mullerian epithelium is established in the ovary over time, in the form of endosalpingiosis, cortical inclusions or endometriosis (figure 4, right). ${ }^{26}$ This epithelium could originate from the tube via exfoliation of tubal epithelial cells or tubal-ovarian adhesions (endosalpingiosis), or via Mullerian metaplasia in the ovarian surface mesothelium. 56 The Mullerian inclusion epithelium itself, or an intervening benign ovarian neoplasm arising in the inclusions, would serve as the epithelial source for the serous carcinoma (figure 3 , left pathway). ${ }^{57}$ In a variation of this pathway, Mullerian epithelium deposited on, or developing in, the pelvic mesothelium would serve as a source of occasional tumors arising in the peritoneum. The second proposed pathway entails malignant transformation of the distal fallopian tube mucosa, initiating as TIC within a pre-existing normal appearing but probably genetically altered epithelium. In this setting, subclones of TIC would evolve by invading underlying stroma, exfoliating to the ovarian (and peritoneal) surfaces, or both, as seen in the cases illustrated in this review (figure 4, left). Instances in which remote metastases were more extensive than the tumor of origin are common and have been reported in preinvasive serous carcinomas of the endometrium. ${ }^{58}$ Like their counterparts in the endometrium, TICs apparently have the capacity to spread beyond the site(s) of origin (i.e., fimbria) with minimal invasion locally, a property that is unique to 
early serous carcinomas. In this scenario, the proximity of the distal fallopian tube to the ovarian surface or peritoneal cavity facilitates early spread of more aggressive tumor clones, which grow at a greater rate in these remote sites.

The above pathways and the assignment of an origin for a given serous carcinoma are a function of five distinct variables. These variables are also outlined in figure 4 .

1. The location of the epithelium at risk. Not all women develop ovarian epithelial inclusions or endometriosis, and if they do, they vary considerably in extent. Inflammatory factors promoting adhesions between tube and ovary and/or the adherences of tubal epithelium to the ovarian surface likewise vary according to the individual. Thus, the location of the susceptible epithelium, and the ultimate origin of a given tumor, may be subject to events that have no direct relationship to carcinogenesis.

2. A genotoxic stress that promotes genetic alterations. The candidate mechanism is oxidative injury, perhaps a function of ovulation. Because ovulation occurs on the ovarian surface, both the OSE and the distal tube (fimbria) would be exposed. ${ }^{59}$ Factors promoting or inhibiting ovulation (e.g., pregnancies, contraceptives) would influence cancer risk. ${ }^{29}$

3. A susceptible epithelial target. Target cell type likely plays a major role in tumorigenesis and the molecular pathways that are involved can be cell type-specific. The evolution of endometrioid, low grade serous and mucinous tumors of the ovarian cortex involves a series of molecular events that are distinct from serous carcinoma and appear to evolve from a cell lineage pathways other than the pure tubal secretory cell (M Parast, CP Crum, MS Hirsch, unpublished data). ${ }^{20}$

4. Genetic risk factors. BRCA mutations and other genetic factors would increase vulnerability to genotoxic injury and/or accelerate the transition from genotoxic injury to neoplasia.

5. Tumor evolution. Serous carcinomas vary in their aggressiveness. Some tend to be confined to peritoneal surfaces, others deeply invade tissue and still others are prone to vascular invasion. This variable would have considerable impact on perceptions of tumor origin. For example, a small tumor arising in the tubal fimbria that rapidly spread and metastasized would be viewed as a primary ovarian or peritoneal tumor. In contrast, a more slowly growing tumor that remained in the fallopian tube would be classified as primary tubal. Yet, the difference between the two perceptions could be as simple as the velocity with which the tumor evolved and metastasized. Similar parallels can be seen with other tumor systems, wherein the primary neoplasm remains small as the more aggressive components of the tumor spread to other sites and preferentially expand.

Table 2. A unifying model for ovarian and pelvic epithelial cancer.

\begin{tabular}{|c|c|c|}
\hline & Pathway 1 & Pathway 2 \\
\hline Target cell & Modified secretory and ciliated & Secretory ${ }^{61}$ \\
\hline Source & $\begin{array}{l}\text { Mullerian metaplasia on ovarian or peritoneal } \\
\text { surfaces, tubal transport (endosalpingiosis) }\end{array}$ & $\begin{array}{l}\text { Tubal mucosa or tubal transport } \\
\text { (endo-salpingiosis) to ovary or } \\
\text { peritoneum }{ }^{21}\end{array}$ \\
\hline Phenotypic range & Broad & Narrow \\
\hline Initial event & $\begin{array}{l}\text { Changes in epithelial phenotype } \\
\text { (endometriosis, mucinous metaplasia) } \\
\text { linked to homeobox dysregulation }{ }^{21}\end{array}$ & $\begin{array}{l}\text { Genotoxic stress with DNA damage } \\
(\text { Ovulation? })^{55}\end{array}$ \\
\hline Gene mutations & $\begin{array}{l}\text { K-ras, B-RAF, PTEN, beta-catenin (Type I) } \\
\text { and p53 (Type II) }\end{array}$ & p53 (Type II) ${ }^{18}$ \\
\hline Early lesion & Cystadenoma, borderline cystadenoma ${ }^{18}$ & p53 signature (latent precancer) ${ }^{55}$ \\
\hline Malignancy & $\begin{array}{l}\text { Endometrioid, clear cell, mucinous, } \\
\text { serous (less common) carcinoma }\end{array}$ & $\begin{array}{l}\text { Intraepithelial, invasive and metastatic } \\
\text { serous carcinoma }\end{array}$ \\
\hline Transition to malignancy & Gradual & Abrupt \\
\hline Most common spread pattern & $\begin{array}{l}\text { Multifocal distribution, distant recurrence, } \\
\text { systemic spread }\end{array}$ & $\begin{array}{l}\text { Mesothelial lined surfaces of the ovary, } \\
\text { fallopian tube, peritoneum }\end{array}$ \\
\hline
\end{tabular}


Applications to Pelvic Serous Cancer Prophylaxis and Prevention

Prophylaxis in the Cancer-prone Individual

The current management of the BRCA+ woman entails a complete examination of the ovaries and tubes. In many practices, the discovery of an early cancer (i.e., intraepithelial carcinoma) will result in a course of chemotherapy, even if the peritoneal washings are negative for tumor cells. Two important responsibilities include maximizing the chance that an occult carcinoma will be recognized and avoiding the misclassification of benign conditions as early cancer. Careful examination of the fimbriae is a necessary component of this process. Early tumors can be identified in a single tubal plica and thorough examination with the SEE-FIM or similar protocol is recommended. The second responsibility, ensuring that a candidate early cancer is accurately diagnosed, falls with the pathologist who must correctly apply the criteria for early serous carcinomas. Recently, pathologists have found that immunostaining suspicious areas for both p53 and MIB-1 (a proliferative marker that will be highly expressed in carcinomas) is helpful in confirming a diagnosis of early carcinoma. ${ }^{50,51,52}$ The importance of proper diagnosis cannot be overstated, given the psychological and clinical implications of such a diagnosis.

An obvious question that arises with the emerging data in $\mathrm{BRCA}+$ women is whether a prophylactic salpingectomy would suffice to prevent ovarian cancer. The implications of this question, both in terms of ovarian preservation and risk, are significant. Based on table 1 and the presence of alternative pathways of cancer development (figure 4), it would be ill advised to assume that prophylactic salpingectomy would prevent all carcinomas from developing in BRCA+ women. However, based on the observations that many of the early carcinomas in this population arise in the distal fallopian tube, systematic examination of the fimbria in larger prospective studies are needed to establish precisely the origin(s) (e.g., tube versus ovary) of early serous carcinomas in BRCA+ individuals.

\section{Future Directions in Ovarian Cancer Prevention}

If this proposed model (table 2) is correct, biomarker development holds considerable promise for the early detection of tumors that arise from ovarian cortical inclusions or endometriosis, many in the so-called Type I pathway. ${ }^{18,19} \mathrm{~A}$ prime candidate for prevention research is endometriosis, for which new animal models have recently been developed. ${ }^{19}$ In contrast, it is likely that a significant proportion of pelvic serous carcinomas will be very difficult to prevent by detection strategies that use biomarkers expressed in malignant cells. However, these discoveries raise considerable hope for approaches designed to reduce the risk of precursor development and/or removal of the epithelium that is most susceptible to early serous carcinoma. To this end, greater knowledge of the role of oxidative injury in early carcinogenesis, or of how risk-lowering variables such as oral contraceptive therapy impact on both ovarian surface and tubal epithelium, is important. ${ }^{60}$ Like the cervix, the road to preventing pelvic serous carcinomas may be paved by a better understanding of candidate precursors, including p53 signatures that arise in the distal fallopian tube. As in the cervix, early serous precursors likely are common and most will not progress. Nevertheless, their prevention could be one important key to ultimately preventing this subset of ovarian epithelial carcinomas.

\section{Acknowledgments}

The authors thank the faculty and resident staff in the Division of Women's and Perinatal Pathology, Department of Pathology at Brigham and Women's Hospital for their assistance with the SEE-FIM protocol. We also thank Dr. Donald Goldstein, Dr. Daniel Cramer and Ms. Kathleen Mitchell for their support.

\section{References}

1. Quirk JT, Natarajan N, Mettlin CJ. Age-specific ovarian cancer incidence rate patterns in the United States. Gynecol Oncol 2005;99:248-250.

2. Armstrong DK, Bundy B, Wenzel L, Huang HQ, Baergen R, Lele S, Copeland LJ, Walker JL, Burger RA; Gynecologic Oncology Group. Intraperitoneal cisplatin and paclitaxel in ovarian cancer. N Engl J Med 2006;354:34-43.

3. Palma M, Ristori E, Ricevuto E, Giannini G, Gulino A. BRCA1 and BRCA2: the genetic testing and the current management options for mutation carriers. Crit Rev Oncol Hematol 2006;57:1-23.

4. Lux MP, Fasching PA, Beckmann MW. Hereditary breast and ovarian cancer: review and future perspectives. J Mol Med 2006;84:16-28.

5. Sogaard M, Kjaer SK, Gayther S. Ovarian cancer and genetic susceptibility in relation to the BRCA1 and BRCA2 genes. Occurrence, clinical importance and intervention. Acta Obstet Gynecol Scand 2006;85:93-105.

6. Olopade OI, Artioli G. Efficacy of risk-reducing salpingo-oophorectomy in women with BRCA-1 and BRCA-2 mutations. Breast J 2004;10:S5-S9.

7. Scully RE, Young RH, Clement PB. Atlas of tumor pathology: tumors of the ovary, maldeveloped gonads, fallopian tube, and broad ligament. $3^{\text {rd }}$ series. Washington, DC: Armed Forces Institute of Pathology; 1996. 51-128.

8. Kabawat SE, Bast RC Jr, Bhan AK, Welch WR, Knapp RC, Colvin $\mathrm{RB}$. Tissue distribution of a coelomic-epithelium-related antigen recognized by the monoclonal antibody OC125. Int $\mathbf{J}$ Gynecol Pathol 1983;2:275-285.

9. Bast RC Jr, Badgwell D, Lu Z, Marquez R, Rosen D, Liu J, Baggerly KA, Atkinson EN, Skates S, Zhang Z, Lokshin A, Menon U, Jacobs I, Lu K. New tumor markers: CA125 and beyond. Int J Gynecol Cancer 2005;15:274-281.

10. Drapkin R, von Horsten HH, Lin Y, Mok SC, Crum CP, Welch WR, Hecht JL. Human epididymis protein 4 (HE4) is a secreted glycoprotein that is overexpressed by serous and endometrioid ovarian carcinomas. Cancer Res 2005;65:2162-2169.

11. Schorge JO, Drake RD, Lee H, Skates SJ, Rajanbabu R, Miller DS, Kim JH, Cramer DW, Berkowitz RS, Mok SC. Osteopontin as an adjunct to CA125 in detecting recurrent ovarian cancer. Clin Cancer Res 2004;10:3474-3478.

12. Rosen DG, Wang L, Atkinson JN, Yu Y, Lu KH, Diamandis EP, Hellstrom I, Mok SC, Liu J, Bast RC Jr. Potential markers that complement expression of CA125 in epithelial ovarian cancer. Gynecol Oncol 2005;99:267-277.

13. Sharma A, Menon U. Screening for gynaecological cancers. Eur J Surg Oncol 2006;32:818-824. 
14. Zhang Z, Barnhill SD, Zhang H, Xu F, Yu Y, Jacobs I, Woolas RP, Berchuck A, Madyastha KR, Bast RC Jr. Combination of multiple serum markers using an artificial neural network to improve specificity in discriminating malignant from benign pelvic masses. Gynecol Oncol 1999;73:56-61.

15. Fields MM, Chevlen E. Ovarian cancer screening: a look at the evidence. Clin J Oncol Nurs 2006;10:77-81.

16. Katabuchi H, Okamura H. Cell biology of human ovarian surface epithelial cells and ovarian carcinogenesis. Med Electron Microsc 2003;36:74-86.

17. Drapkin RL, Hecht JL. Pathogenesis of ovarian cancer. In: Crum CP, Lee KR, eds. Diagnostic gynecologic and obstetric pathology. Philadelphia, PA: WB Saunders; 2006. 793-810.

18. Singer G, Stohr R, Cope L, Dehari R, Hartmann A, Cao DF, Wang TL, Kurman RJ, Shih IeM. Patterns of p53 mutations separate ovarian serous borderline tumors and low- and high-grade carcinomas and provide support for a new model of ovarian carcinogenesis: a mutational analysis with immunohistochemical correlation. Am J Surg Pathol 2005;29:218-224.

19. Dinulescu DM, Ince TA, Quade BJ, Shafer SA, Crowley D, Jacks T. Role of K-ras and Pten in the development of mouse models of endometriosis and endometrioid ovarian cancer. Nat Med 2005;11:63-70.

20. Cheng W, Liu J, Yoshida H, Rosen D, Naora H. Lineage infidelity of epithelial ovarian cancers is controlled by HOX genes that specify regional identity in the reproductive tract. Nat Med 2005;11:531-537.

21. Marquez RT, Baggerly KA, Patterson AP, Liu J, Broaddus R, Frumovitz M, Atkinson EN, Smith DI, Hartmann L, Fishman D, Berchuck A, Whitaker R, Gershenson DM, Mills GB, Bast $\mathrm{RC} \mathrm{Jr}, \mathrm{Lu} \mathrm{KH}$. Patterns of gene expression in different histotypes of epithelial ovarian cancer correlate with those in normal fallopian tube, endometrium, and colon. Clin Cancer Res 2005;11:6116-6126.

22. Leitao MM, Soslow RA, Baergen RN, Olvera N, Arroyo C, Boyd J. Mutation and expression of the TP53 gene in early stage epithelial ovarian carcinoma. Gynecol Oncol 2004;93:301-306.

23. Bell DA. Origins and molecular pathology of ovarian cancer. Mod Pathol 2005;18:S19-S32.

24. Aoki Y, Kawada N, Tanaka K. Early form of ovarian cancer originating in inclusion cysts. A case report. J Reprod Med 2000;45:159-161.

25. Hutson R, Ramsdale J, Wells M. p53 protein expression in putative precursor lesions of epithelial ovarian cancer. Histopathology 1995;27:367-371.

26. Bell DA, Scully RE. Early de novo ovarian carcinoma. A study of fourteen cases. Cancer 1994;73:1859-1864.

27. Schlosshauer PW, Cohen CJ, Penault-Llorca F, Miranda CR, Bignon YJ, Dauplat J, Deligdisch L. Prophylactic oophorectomy: a morphologic and immunohistochemical study. Cancer 2003;98:2599-2606.

28. Barakat RR, Federici MG, Saigo PE, Robson ME, Offit K, Boyd J. Absence of premalignant histologic, molecular, or cell biologic alterations in prophylactic oophorectomy specimens from BRCA1 heterozygotes. Cancer 2000;89:383-390.

29. Brewer MA, Johnson K, Follen M, Gershenson D, Bast R Jr. Prevention of ovarian cancer: intraepithelial neoplasia. Clin Cancer Res 2003;9:20-30.

30. Richart RM. Cervical intraepithelial neoplasia. Pathol Annu 1973;8:301-328.

31. Crum CP, Ikenberg H, Richart RM, Gissman L. Human papillomavirus type 16 and early cervical neoplasia. N Engl J Med 1984;310:880-883.

32. Koutsky LA, Ault KA, Wheeler CM, Brown DR, Barr E, Alvarez FB, Chiacchierini LM, Jansen KU; Proof of Principle Study Investigators. A controlled trial of a human papillomavirus type 16 vaccine. N Engl J Med 2002;347:1645-1651.
33. Kauff ND, Satagopan JM, Robson ME, Scheuer L, Hensley M, Hudis CA, Ellis NA, Boyd J, Borgen PI, Barakat RR, Norton L, Castiel M, Nafa K, Offit K. Risk-reducing salpingo-oophorectomy in women with a BRCA1 or BRCA2 mutation. N Engl J Med 2002;346:1609-1615.

34. Rebbeck TR, Lynch HT, Neuhausen SL, Narod SA, Van’t Veer L, Garber JE, Evans G, Isaacs C, Daly MB, Matloff E, Olopade OI, Weber BL; Prevention and Observation of Surgical End Points Study Group. Prophylactic oophorectomy in carriers of BRCA1 or BRCA2 mutations. N Engl J Med 2002;346:1616-1622.

35. Rosenblatt KA, Weiss NS, Schwartz SM. Incidence of malignant fallopian tube tumors. Gynecol Oncol 1989;35:236-239.

36. Lee KR, Tabassoli FA, Prat J, Dietel M, Gersell DJ, Karseladze AI, Hauptmann S, Rutgers J, Russell P, Buckley CH, Pisani P, Schwartz P, Goldgar DE, Silva E, Caduff R, Kubik-Huch RA. Surface epithelial-stromal tumours. In: Tavassoli FA, Devilee P, eds. World Health Organization classification of tumours: pathology and genetics: tumours of the breast and female genital organs. Lyon: IARC Press; 2003. 117-145.

37. Piek JM, Kenemans P, Verheijen RH. Intraperitoneal serous adenocarcinoma: a critical appraisal of three hypotheses on its cause. Am J Obstet Gynecol 2004;191:718-732.

38. Zweemer RP, van Diest PJ, Verheijen RH, Ryan A, Gille JJ, Sijmons RH, Jacobs IJ, Menko FH, Kenemans P. Molecular evidence linking primary cancer of the fallopian tube to BRCA1 germline mutations. Gynecol Oncol 2000;76:45-50.

39. Colgan TJ, Boerner SL, Murphy J, Cole DE, Narod S, Rosen B. Peritoneal lavage cytology: an assessment of its value during prophylactic oophorectomy. Gynecol Oncol 2002;85:397-403.

40. Agoff SN, Mendelin JE, Grieco VS, Garcia RL. Unexpected gynecologic neoplasms in patients with proven or suspected BRCA-1 or -2 mutations: implications for gross examination, cytology, and clinical follow-up. Am J Surg Pathol 2002;26:171-178.

41. Colgan TJ, Murphy J, Cole DE, Narod S, Rosen B. Occult carcinoma in prophylactic oophorectomy specimens: prevalence and association with BRCA germline mutation status. Am J Surg Pathol 2001;25:1283-1289.

42. Powell CB, Kenley E, Chen LM, Crawford B, McLennan J, Zaloudek C, Komaromy M, Beattie M, Ziegler J. Risk-reducing salpingo-oophorectomy in BRCA mutation carriers: role of serial sectioning in the detection of occult malignancy. J Clin Oncol 2005;23:127-132.

43. Finch A, Shaw P, Rosen B, Murphy J, Narod SA, Colgan TJ. Clinical and pathologic findings of prophylactic salpingo-oophorectomies in 159 BRCA1 and BRCA2 carriers. Gynecol Oncol 2006;100:58-64.

44. Cass I, Holschneider C, Datta N, Barbuto D, Walts AE, Karlan BY. BRCA-mutation-associated fallopian tube carcinoma: a distinct clinical phenotype? Obstet Gynecol 2005;106:1327-1334.

45. Risch HA, McLaughlin JR, Cole DE, Rosen B, Bradley L, Kwan E, Jack E, Vesprini DJ, Kuperstein G, Abrahamson JL, Fan I, Wong B, Narod SA. Prevalence and penetrance of germline BRCA1 and BRCA2 mutations in a population series of 649 women with ovarian cancer. Am J Hum Genet 2001;68:700-710.

46. Aziz S, Kuperstein G, Rosen B, Cole D, Nedelcu R, McLaughlin J, Narod SA. A genetic epidemiological study of carcinoma of the fallopian tube. Gynecol Oncol 2001;80:341-345.

47. Levine DA, Argenta PA, Yee CJ, Marshall DS, Olvera N, Bogomolniy F, Rahaman JA, Robson ME, Offit K, Barakat RR, Soslow RA, Boyd J. Fallopian tube and primary peritoneal carcinomas associated with BRCA mutations. J Clin Oncol 2003;21:4222-4227. 
48. Alvarado-Cabrero I, Navani SS, Young RH, Scully RE. Tumors of the fimbriated end of the fallopian tube: a clinicopathologic analysis of 20 cases, including nine carcinomas. Int J Gynecol Pathol 1997;16:189-196.

49. Chang P, Crum C. The fallopian tube. In: Crum CP, Lee KR, eds. Diagnostic gynecologic and obstetric pathology. Philadelphia, PA: WB Saunders; 2006.

50. Medeiros F, Muto MG, Lee Y, Elvin JA, Callahan MJ, Feltmate C, Garber JE, Cramer DW, Crum CP. The tubal fimbria is a preferred site for early adenocarcinoma in women with familial ovarian cancer syndrome. Am J Surg Pathol 2006;30:230-236.

51. Lee Y, Medeiros F, Kindelberger D, Callahan MJ, Muto MG, Crum CP. Advances in the recognition of tubal intraepithelial carcinoma: applications to cancer screening and the pathogenesis of ovarian cancer. Adv Anat Pathol 2006;13:1-7.

52. Bannatyne P, Russell P. Early adenocarcinoma of the fallopian tubes. A case for multifocal tumorigenesis. Diagn Gynecol Obstet 1981;3:49-60.

53. Kindelberger DW, Lee Y, Miron A, Hirsch MS, Feltmate C, Medeiros F, Callahan MJ, Garner EO, Gordon RW, Birch C, Berkowitz RS, Muto MG, Crum CP. Intraepithelial carcinoma of the fimbria and pelvic serous carcinoma: evidence for a causal relationship. Am J Surg Pathol 2007;31:161-169.

54. Marquez RT, Baggerly KA, Patterson AP, Liu J, Broaddus R, Frumovitz M, Atkinson EN, Smith DI, Hartmann L, Fishman D, Berchuck A, Whitaker R, Gershenson DM, Mills GB, Bast $\mathrm{RC} \mathrm{Jr}, \mathrm{Lu} \mathrm{KH}$. Patterns of gene expression in different histotypes of epithelial ovarian cancer correlate with those in normal fallopian tube, endometrium, and colon. Clin Cancer Res 2005;11:6116-6126.

55. Lee Y, Miron A, Drapkin R, Nucci MR, Medeiros F, Saleemuddin A, Garber J, Birch C, Mou H, Gordon RW, Cramer DW, McKeon FD, Crum CP. A candidate precursor to serous carcinoma that originates in the distal fallopian tube. J Pathol 2007;211:26-35.

56. Zheng W, Li N, Wang J, Ulukus EC, Ulukus M, Arici A, Liang SX. Initial endometriosis showing direct morphologic evidence of metaplasia in the pathogenesis of ovarian endometriosis. Int J Gynecol Pathol 2005;24:164-172.

57. Korner M, Burckhardt E, Mazzucchelli L. Different proportions of aneusomic cells in ovarian inclusion cysts associated with serous borderline tumours and serous high-grade carcinomas support different pathogenetic pathways. J Pathol 2005;207:20-26.

58. Soslow RA, Pirog E, Isacson C. Endometrial intraepithelial carcinoma with associated peritoneal carcinomatosis. Am J Surg Pathol 2000;24:726-732.

59. Murdoch WJ. Carcinogenic potential of ovulatory genotoxicity. Biol Reprod 2005;73:586-590.

60. Rodriguez GC, Nagarsheth NP, Lee KL, Bentley RC, Walmer DK, Cline M, Whitaker RS, Isner P, Berchuck A, Dodge RK, Hughes CL. Progestin-induced apoptosis in the Macaque ovarian epithelium: differential regulation of transforming growth factor-beta. J Natl Cancer Inst 2002;94:50-60.

61. Rorat E, Fenoglio C. The ultrastructure of a poorly differentiated adenocarcinoma of the human tuba uterina. Oncology 1976;33:167-169.

\section{Author Affiliations}

Christopher P. Crum, MD

Division of Women's and Perinatal Pathology

Department of Pathology

Brigham and Women's Hospital

Boston, MA 02115
Ronny Drapkin, $M D, P h D$

Division of Medical Oncology

Dana Farber Cancer Institute

Boston, MA 02115

David Kindelberger, $M D$

Division of Women's and Perinatal Pathology

Department of Pathology

Brigham and Women's Hospital

Boston, MA 02115

Fabiola Medeiros, MD

Division of Women's and Perinatal Pathology

Department of Pathology

Brigham and Women's Hospital

Boston, MA 02115

Alexander Miron, PhD

Division of Cancer Biology

Dana Farber Cancer Institute

Boston, MA 02115

Yonghee Lee, MD

Division of Women's and Perinatal Pathology

Department of Pathology

Brigham and Women's Hospital

Boston, MA 02115 